\title{
Las creencias irracionales en relación con ciertas conductas de consumo en adolescentes
}

\author{
Miguel Á. Carbonero, Luis J. Martín-Antón y Marta Feijó \\ Universidad de Valladolid (España)
}

\begin{abstract}
Esta investigación se enmarca en un encuadre cognitivo. Considera también el componente físiológico de la conducta, ya sea como elemento de la misma o como posible causa remota, sobre todo en ciertas patologías. Se somete a análisis la incidencia de las creencias irracionales y de aceptación para ver su relación con las anteriores creencias irracionales y el impacto de ambas en las siguientes áreas: consumo de sustancias psicoactivas y percepción medioambiental en los jóvenes. A su vez, la percepción medioambiental es incluida por la importancia de las conductas en favor de la ecología que aquella promueve, necesarias hoy en día tanto en jóvenes como en adultos para la propia sostenibilidad del planeta. Este trabajo constituye una aportación en la búsqueda de las carencias psicológicas y necesidades emocionales de los adolescentes. Todo resultado positivo en esta línea será considerado como demanda de una atención y formación específica en este ámbito que los jóvenes reclaman en su derecho a recibir una educación integral y de calidad. Sería necesario ayudar a las nuevas generaciones a construir libremente su proyecto existencial para que su vida adquiera un nuevo significado.
\end{abstract}

Palabras clave: Creencias irracionales, consumo de drogas, adolescentes.

Irrational beliefs in relation to certain behaviors in adolescents. This research is part of a cognitive frame. Consider also the physiological component of the conduct, whether as part of the same or as a possible remote cause, especially in certain pathologies. Being analyzed the impact of irrational beliefs and acceptance to see their relationship with the previous irrational beliefs and the impact of both in the following areas: consumption of psychoactive substances and environmental awareness in youth. In turn, environmental awareness is included for the importance of behavior in favor of ecology that promotes that are necessary today in both youth and adults for self-sustainability of the planet. This work constitutes a contribution in the search for psychological deficits and emotional needs of adolescents. Any positive result in this line will be considered as a demand for attention and specific training in this area that young people claim their right to receive comprehensive and quality education. Would be necessary to help new generations to build freely existential project that his life acquired a new meaning.

Key words: Irrational beliefs, drug use, adolescents.

Correspondencia: Miguel Ángel Carbonero Martín. Universidad de Valladolid. Departamento de Psicología. Facultad de Educación y Trabajo Social. Campus Miguel Delibes. Paseo de Belén, s/n. C.P. 47011. Valladolid (España). E-mail: carboner@psi.uva.es 
La Organización Mundial de la Salud (OMS) delimita la adolescencia como un período de edad comprendido entre los 11 y los 19 años. La realidad humana que abarca este concepto no puede considerarse, sin embargo, uniforme, pues depende -entre otros factores- del grupo social que se considere. De acuerdo con Corral y Pardo (2001), la adolescencia debe ser abordada desde un enfoque multidisciplinar que tenga en cuenta los diferentes contextos históricos y culturales, los cambios individuales tanto a nivel psicológico como biológico, y las interacciones grupales e interpersonales. Para comprender el período adolescente de la forma más completa y objetiva posible no deben olvidarse, por tanto, dos perspectivas esenciales: los distintos enfoques teóricos desde los que se afronta el estudio del desarrollo humano y las diferentes circunstancias desde las que los propios protagonistas se enfrentan a este período vital.

Durante la adolescencia se producen cambios neurofisiológicos y biográficos que influyen en la evolución de la actividad mental y ésta, a su vez, en el resto de funciones y comportamientos de nueva adquisición. Pese a la heterogeneidad de este período, diversos autores destacados en el ámbito de la Psicología evolutiva han extraído características comunes a los individuos de estas edades. Desde teorías organísmicas clásicas, Inhelder y Piaget (1955) proponen un nuevo estadio de desarrollo cognitivo, el pensamiento formal, que aparecería entre los 11 y 12 años, para consolidarse posteriormente. El adolescente comienza a manejar un pensamiento más abstracto que le permite seguir una secuencia de razonamiento hipotético-deductiva. Aunque con un desarrollo incompleto aún a nivel metacognitivo, la capacidad de concebir lo posible empuja al adolescente hacia el reto de construir una identidad de sí mismo coherente, remarcada desde posiciones psicoanalíticas (Erikson, 1968). Es lo que Bruner (1966) llamó también lógica narrativa, como desarrollo dentro del ámbito del pensamiento no formal. El razonamiento moral también experimenta cambios en la adolescencia. Kohlberg (1984) propone la posibilidad de adquisición de una moral postconvencional basada en principios más universales y no tanto culturales. Por último, Rosenberg (1965) remarca el cambio en el autoconcepto del adolescente que se hace más interno, autónomo, complejo y abstracto respecto a etapas anteriores.

El ambiente cultural y familiar proporciona los contenidos cognitivos que todo niño y, posteriormente adolescente, asume como creencias propias, filosofías de vida, esquemas cognitivos, etc. Desde un ámbito más clínico de la Psicología se ha abordado el pensamiento como causante de la conducta y la afectividad. Esto es así concretamente en las terapias racionales y de reestructuración cognitiva, la pionera de las cuales fue la Terapia Racional Emotiva fundada por Ellis a comienzos de los setenta. Ellis adopta el llamado "esquema A-B-C" a través del cual explica que las consecuencias emocionales y conductuales, $\mathrm{C}$, no son provocadas directamente por los acontecimientos activadores, A, sino por las creencias de la persona acerca de A, es decir, B. 
Las creencias pueden ser expresadas en forma deseos o preferencias -lo que Ellis (1981) denominó creencias racionales- o como una obligación, necesidad imperiosa o exigencia. Estas últimas serían creencias irracionales, manifestaciones dogmáticas y absolutas cuya no consecución provoca emociones negativas inadecuadas y alteraciones de conducta que interfieren en la obtención de nuevas metas. Aunque las creencias irracionales que mantiene la gente pueden ser infinitas, Ellis las agrupa en tres peticiones de carácter absoluto que puede manifestar el individuo: "tengo que actuar bien y ganar la aprobación por ello", "tú debes actuar de forma agradable y justa conmigo" y "mi vida debe ser lo suficientemente buena y fácil para conseguir lo que quiero sin demasiado esfuerzo". Los objetivos fundamentales de la Terapia Racional Emotiva son, como dice Carrasco-Galán (2005), conseguir en el individuo la autoaceptación y la tolerancia a la frustración. Dos objetivos que, superficialmente, parecerían estar relacionados con un nuevo enfoque terapéutico aparecido hace tan sólo una década: la Terapia de Aceptación y Compromiso.

La Terapia de Aceptación y Compromiso, ACT, (Hayes, Stroshal y Wilson, 1999) es considerada por Luciano y Valdivia (2006), como la más completa de las Terapias de Conducta de Tercera Generación. La primera y segunda generaciones de terapias corresponderían a los paradigmas conductista y cognitivo respectivamente, según la clasificación de Hayes (2004). La ACT se enmarca en una filosofía contextualfuncional -la Teoría del Marco Relacional- que concibe la historia personal como inductora de los contenidos y esquemas cognitivos privados. Sin embargo, califica como relaciones arbitrarias potenciadas socialmente a aquéllas que se producen entre dichos eventos privados y las acciones del organismo. Esta afirmación sitúa a la ACT en desacuerdo con las terapias cognitivo-conductuales a las que critica por falta de base experimental, pues no llega a conocerse realmente la causa del éxito o fracaso del tratamiento.

La ACT recalca la dualidad placer-sufrimiento de la vida humana y observa que las culturas "avanzadas" viven buscando el placer inmediato y evitando el dolor. Un patrón de conducta inflexible y extremo en este sentido llevaría a la clasificación del individuo en una categoría patológica global y no reduccionista llamada Trastorno de Evitación Experiencial (Hayes, Wilson, Gifford, Follete y Strosahl, 1996; Luciano y Hayes, 2001). La Evitación Experiencial supone una necesidad de controlar y/o evitar la presencia de pensamientos, recuerdos, sensaciones y otros eventos privados que provocan malestar. Paradójicamente, la continua necesidad de evitar el sufrimiento y obtener placer inmediato para vivir obligan a la persona a actuar de modo que no "vive". Dichos comportamientos proporcionan un relativo alivio inmediato en ocasiones, pero provocan que, a más largo plazo, se reviva el malestar incluso de manera más intensa y duradera. Esto "obliga" al individuo a seguir intentando eliminar el sufrimiento que, a su vez, estará cada vez más presente. Al final el tiempo se reduce a hacer cosas para que 
desaparezca el malestar, y el resultado es un abandono de las acciones potencialmente valiosas.

Parece, en conclusión, que tanto las consecuencias de mostrar un patrón de evitación experiencial como las de albergar en la mente creencias irracionales se manifiestan a nivel general en la vida del individuo en forma de acciones, afectos o cogniciones. Y por individuo no sólo se entiende la persona adulta, sino que también los adolescentes pueden mostrar niveles desadaptativos o patológicos de evitación experiencial y creencias irracionales que influyan negativamente en las diferentes facetas de su vida. Para comprobar dicha influencia y correlación se han elegido tres variables que abarcan el ocio juvenil: la relación con el medio ambiente y el consumo de sustancias. La relación de estos factores con las creencias o la aceptación apenas posee bibliografía publicada al respecto por lo que el encuadre teórico se centra principalmente en la descripción de cada variable en particular.

Desde mediados de los 70 ya se planteaba que las creencias que la sociedad mantenía sobre su relación con el medio ambiente conformaban el modo en que ésta conceptualizaba la Naturaleza y se comportaba frente a ella. Dunlap y Van Liere (1984) proponen el surgimiento de un nuevo paradigma de tipo ecocéntrico, frente al antropocentrismo dominante. En nuestro país, Amérigo y González (2000) han adaptado al español la escala de Dunlap sobre creencias generales acerca de la relación del ser humano con el medio ambiente (Escala NEP: Nuevo Paradigma Ecológico). Más recientemente, Vozmediano y San Juan (2005) analizan las propiedades psicométricas de esta escala en una muestra española a través de Internet.

Además del interés por averiguar si los jóvenes españoles se preocupan por la salud del planeta, sigue siendo un reto pendiente el mejorar sus propios hábitos de salud, sobre todo en lo que se refiere al consumo de sustancias. Los resultados a la encuesta sobre el uso de drogas en población escolar (14-18 años) publicada por el Observatorio Español sobre Drogas en el año 2006 muestran una disminución, respecto a años anteriores, en la prevalencia del consumo de la mayoría de las sustancias. Las drogas mayormente consumidas por los jóvenes siguen siendo el alcohol, el tabaco y el cannabis. Un dato significativo es la sustancial diferencia en un mayor consumo de aquellos adolescentes cuyos amigos son también consumidores, con respecto a aquéllos cuyo grupo de amigos mayoritariamente no consume. Esto conduce, sin duda, a valorar la enorme importancia que tiene la investigación acerca de los factores que pueden estar determinando el origen y mantenimiento del consumo. Aunque, como sostienen Holden, Moncher y Schinke (1990) respecto al abuso de alcohol, ninguna teoría ha tenido una aceptación clara.

Hidalgo y Redondo (2005) detallan en un artículo la diversidad de factores estudiados asociados a la experimentación y el abuso de sustancias, desde los socioculturales y ambientales -como la norma social permisiva- hasta los biológicos 
como el sexo o la genética. La familia, el entorno educativo o el grupo de iguales también serían importantes variables a considerar. En relación a los objetivos de este estudio se remarcan los factores psicológicos, como las creencias. En concreto, las creencias irracionales se han demostrado predictores del abuso de sustancias en los jóvenes (Denoff, 2006; Camatta y Naghosi, 1995). Pero hay que tener también en cuenta estudios que muestran datos en contra de lo que cabría pensar respecto a las variables asociadas al consumo. Por ejemplo, Pastor, Balaguer y García-Merita (2006) encuentran que la adecuación conductual, la aceptación social y la amistad íntima serían los mejores predictores de conductas de riesgo para la salud en la población juvenil, así como las creencias motivacionales pueden ser un factor protector del estrés (González-Cabanach, Valle, Rodríguez, Piñeiro y González, 2010). Por último, es necesario remarcar la diferencia entre consumo y abuso. Aunque posiciones radicales consideran abuso cualquier consumo por parte de un menor, la categorización de un consumo como abusivo dependería de los patrones de consumo y las consecuencias negativas asociadas a los mismos.

\section{METODO}

\section{Participantes}

Han participado 208 adolescentes de entre 14 y 19 años, con una media de 16.02 años $(D T=1.183)$. La mayoría está entre los 15 y 17 años $(81.2 \%)$, escolarizados en los dos últimos cursos de Educación Secundaria Obligatoria y Bachillerato de varios Institutos de Educación Secundaria de Valladolid (Tabla 1). En cuanto al sexo, el 51\% son mujeres y el $49 \%$ varones.

\begin{tabular}{lcc}
\multicolumn{3}{c}{ Tabla 1. Distribución por Curso } \\
\hline Curso & Frecuencia & Porcentaje \\
\hline $3^{\circ}$ ESO & 35 & 16.8 \\
$4^{\circ}$ ESO & 95 & 45.7 \\
$1^{\circ}$ Bachillerato & 56 & 26.9 \\
$2^{\circ}$ Bachillerato & 22 & 10.6 \\
Total & 208 & 100.0 \\
\hline
\end{tabular}

\section{Instrumentos y variables}

El TCI-A Inventario de Creencias Irracionales para adolescentes, fue elaborado por Cardeñoso y Calvete (2004) para evaluar las creencias irracionales en la población adolescente. Consta de 37 ítems a los que se contesta en un grado de acuerdo del 1 al 4. El inventario se compone de una escala de Irracionalidad General (IG) y 6 subescalas: Necesidad de Aceptación y Éxito (NAE), Indefensión (IND), Culpabilización (CUL), Evitación de Problemas (EP), Intolerancia a la Frustración (IF) y Justificación de la Violencia (JV).

El Acceptance and Action Questionnaire (Hayes et al., 2000) evalúa la evitación experiencial y la aceptación psicológica, dos conceptos clave dentro de la 
Terapia de Aceptación y Compromiso. Barraca (2004) ha llevado a cabo la adaptación española del, traducido al castellano, Cuestionario de Aceptación y Acción (AAQ). Consta de 9 ítems que deben contestarse en una escala tipo Likert. Posee un alpha de Cronbach de .74 y una estabilidad temporal de .71 (test-retest). La Actitud Evitativa (AE) está formada por tres subfactores: desbordamiento emocional, inadecuada evaluación de los problemas vitales, y respuesta ineficiente a los problemas vitales.

La Escala Nuevo Paradigma Ecológico (Vozmediano y San Juan, 2005) es una versión española de la escala New Ecological Paradigm (NEP) (Dunlap, Van Liere, Merting y Jones, 2000). Estudia las creencias sobre la Naturaleza y las relaciones entre el ser humano y el medio ambiente. Está formada por 11 ítems a los que se responde mediante una escala tipo Likert (de 0 a 10). Está formada por dos dimensiones, el antropocentrismo (ANTR) y el egocentrismo (ECO), que explican un $23 \%$ y un $22 \%$ de la varianza respectivamente, con un alpha de Cronbach de .71 en ambas escalas.

\section{Procedimiento}

Una vez concretados los objetivos y las hipótesis de trabajo, se seleccionaron los instrumentos más adecuados, por sus características, para medir las diferentes variables. A su vez, los centros fueron elegidos por su representatividad y se estableció el contacto con los mismos. Los cuestionarios fueron pasados por los tutores o profesores que se ofrecieron a dedicar parte de su hora de clase (sólo en algunos casos coincidió con la hora de tutoría). Posteriormente se realizaron análisis de descriptivos, correlaciones y diferencias entre medias mediante el paquete estadístico SPSS v.15.

\section{RESULTADOS}

El primer objetivo es analizar la relación entre las creencias irracionales y la aceptación de los adolescentes en cuanto a su preocupación por del medio ambiente -ecocentrismo-, en contraposición al antropocentrismo, que no ve ninguna obligación moral respecto al cuidado del medio ambiente. El Antropocentrismo correlaciona significativamente con la Evitación de Problemas $(r=.225, p=.002) \mathrm{y}$, en menor medida -aunque también de manera significativa-, con la Necesidad de Aceptación y Éxito $(r=.143, p=.048)$ y la Irracionalidad total $(r=.160, p=.042)$. El Ecocentrismo correlaciona negativamente con la Evitación de Problemas $(r=-.186, p=.010)$, al igual que con la Justificación de la Violencia ( $r=-.202, p=.005)$. Por el contrario, correlaciona significativamente, y de manera positiva, con la Culpabilización ( $r=.229, p=.001)$. También la correlación es significativa, pero en sentido negativo.

Otro aspecto de interés es la relación entre las Creencias Irracionales y de Aceptación. La Irracionalidad total de la muestra correlaciona significativamente con la Actitud Evitativa -en definitiva, lo contrario a la Aceptación- $(r=.309, p=.000)$. Analizando dicha correlación por subfactores es de destacar la mayor relación existente 
entre dicha Irracionalidad total y el subfactor del test de Aceptación Desbordamiento emocional ( $r=.340, p=.000)$. La Actitud Evitativa, a su vez, correlaciona de manera significativa con los subfactores siguientes del Cuestionario de Creencias Irracionales: Necesidad de Aceptación y Éxito ( $r=.348, p=.000)$, Culpabilización $(r=.193, p=.007)$ e Intolerancia a la Frustración ( $r=.255, p=.000)$. A su vez, también tiene una relación significativa, pero menor con la dimensión de Indefensión $(r=.176, p=.014)$. También se produce una correlación significativa y positiva entre Desbordamiento emocional y Necesidad de Aceptación y Éxito ( $r=.364, p=.000)$. El subgrupo de creencias irracionales Justificación de la Violencia se muestra independiente a la Actitud Evitativa, así como, de manera sorprendente, también le ocurre lo mismo al subfactor Evitación de Problemas. Otras correlaciones se producen entre la Respuesta ineficiente a los problemas vitales con la Culpabilización irracional ( $r=.162, p=.021)$.

Por otra parte, en cuanto a la relación entre la Terapia de Aceptación y Compromiso con los paradigmas ecocéntrico y antropocéntrico. No existen correlaciones significativas entre la Actitud Evitativa -ni ninguno de sus subfactores- y el ecocentrismo o el antropocentrismo, excepto entre el Desbordamiento Emocional y el Ecocentrismo $(r=.158, p=.026)$.

En cuanto a las diferencias en las variables anteriormente mencionadas en función del consumo o no de sustancias (Tabla 2), únicamente se obtienen diferencias estadísticamente significativas con el factor de creencias irracionales "indefensión".

Tabla 2. Diferencias en las creencias irracionales y aceptación en función de haber probado o no alguna droga ilegal

\begin{tabular}{|c|c|c|c|c|c|c|c|}
\hline & ¿Ha probado? & $N$ & $M$ & $D T$ & $t$ & $U$ & Sig. \\
\hline \multirow{2}{*}{ NAE } & $\mathrm{Si}$ & 48 & 17.12 & 4.89 & & \multirow{2}{*}{3485.5} & \multirow{2}{*}{.689} \\
\hline & No & 151 & 16.82 & 4.23 & & & \\
\hline \multirow{2}{*}{ IND } & $\mathrm{Si}$ & 47 & 17.80 & 3.01 & & \multirow{2}{*}{$2827.0^{*}$} & \multirow{2}{*}{.034} \\
\hline & No & 151 & 16.81 & 2.79 & & & \\
\hline \multirow{2}{*}{ CUL } & $\mathrm{Si}$ & 51 & 13.64 & 2.65 & \multirow{2}{*}{-0.798} & & \multirow{2}{*}{.426} \\
\hline & No & 152 & 14.00 & 2.82 & & & \\
\hline \multirow{2}{*}{$\mathrm{EP}$} & $\mathrm{Si}$ & 50 & 7.80 & 2.24 & & \multirow{2}{*}{3518.5} & \multirow{2}{*}{.649} \\
\hline & No & 147 & 7.63 & 1.92 & & & \\
\hline \multirow{2}{*}{ IF } & $\mathrm{Si}$ & 53 & 7.52 & 1.87 & & \multirow{2}{*}{3683.5} & \multirow{2}{*}{.462} \\
\hline & No & 149 & 7.27 & 1.97 & & & \\
\hline \multirow{2}{*}{ JV } & $\mathrm{Si}$ & 47 & 15.80 & 4.65 & & \multirow{2}{*}{2885.5} & \multirow{2}{*}{.100} \\
\hline & No & 146 & 14.59 & 4.02 & & & \\
\hline \multirow{2}{*}{ IG } & $\mathrm{Si}$ & 39 & 79.61 & 12.39 & \multirow{2}{*}{1.118} & & \multirow{2}{*}{.265} \\
\hline & No & 130 & 77.44 & 10.04 & & & \\
\hline \multirow{2}{*}{$\mathrm{DE}$} & $\mathrm{Si}$ & 53 & 21.81 & 5.60 & \multirow{2}{*}{-0.319} & & \multirow{2}{*}{.750} \\
\hline & No & 152 & 22.05 & 4.58 & & & \\
\hline \multirow{2}{*}{ IEPV } & $\mathrm{Si}$ & 53 & 9.22 & 2.20 & & \multirow{2}{*}{3767.5} & \multirow{2}{*}{.438} \\
\hline & No & 153 & 8.96 & 2.10 & & & \\
\hline \multirow{2}{*}{ RIPV } & $\mathrm{Si}$ & 54 & 8.01 & 2.64 & & \multirow{2}{*}{4101.5} & \multirow{2}{*}{.881} \\
\hline & No & 154 & 8.22 & 2.19 & & & \\
\hline \multirow{2}{*}{$\mathrm{AE}$} & $\mathrm{Si}$ & 52 & 39.09 & 7.39 & \multirow{2}{*}{-0.178} & & \multirow{2}{*}{.859} \\
\hline & No & 151 & 39.27 & 5.97 & & & \\
\hline
\end{tabular}


Por último, analizamos las diferencias existentes en cada una de las variables contempladas en función de la frecuencia de consumo de sustancias legales. En este sentido, no existen diferencias en cuanto al consumo de cigarrillos y licores. Sin embargo, no ocurre lo mismo con el consumo de cerveza, vino y combinados. En el primer caso (Tabla 3), nos encontramos con diferencias estadísticamente significativas en las variables Culpabilización $(K=9.982, p=.019)$ y Justificación de la Violencia $(K=10.239, p=.017)$ se relacionan significativamente con el hábito de beber cerveza. En el caso de la Justificación de la Violencia es significativamente más alta la puntuación en sujetos que beben más de 150 unidades al mes, con respecto a aquellos que no beben o toman menos de 50 .

Tabla 3. Diferencias en las creencias irracionales y aceptación en función del consumo de cervezas

\begin{tabular}{llccccc}
\hline & Consumo & $N$ & $M$ & $D T$ & $K$ & Sig. \\
\hline \multirow{4}{*}{ CUL } & Ninguno & 128 & 14.21 & 2.81 & & \\
& De 1 a 50 & 43 & 13.62 & 2.73 & & \\
& De 51 a 100 & 16 & 14.50 & 2.16 & $9.982^{*}$ & .019 \\
& De 101 a 150 & 0 & 0.0 & 0.0 & & \\
& Más de 150 & 10 & 11.80 & 1.54 & & \\
\multirow{4}{*}{ JV } & Ninguno & 120 & 14.49 & 3.49 & & \\
& De 1 a 50 & 42 & 14.14 & 4.42 & & \\
& De 51 a 100 & 16 & 16.12 & 5.22 & $10.239 *$ & \\
& De 101 a 150 & 10 & 18.20 & 4.36 & & \\
\hline$* p<.05, * * p<.01, * * * p<.001$ & 188 & 14.75 & 4.01 & &
\end{tabular}

El consumo de vino en los adolescentes, según los resultados de la prueba de Kruskal-Wallis (Tabla 4), no se relaciona con las creencias irracionales. Sin embargo, en referencia a la variable aceptación, presenta una relación significativa con el factor Respuesta ineficiente a los problemas vitales $(K=12.995, p=.011)$. La diferencia de medias es más acusada entre las personas que no beben vino o beben menos de 50 vasos al mes y los que beben entre 50 y 100 vasos mensuales.

Tabla 4. Diferencias en las creencias irracionales y aceptación en función del consumo de vino

\begin{tabular}{llccccc}
\hline & Consumo & $N$ & $M$ & $D T$ & \multirow{2}{*}{ S } & Sig. \\
\hline \multirow{4}{*}{ RIPV } & Ninguno & 155 & 8.38 & 2.12 & & \\
& De 1 a 50 & 28 & 8.53 & 1.99 & & \\
& De 51 a 100 & 9 & 5.11 & 3.14 & $12.995 *$ & .011 \\
& De 101 a 150 & 2 & 7.50 & 0.70 & & \\
Más de 150 & 8 & 6.25 & 3.41 & & \\
\hline$p<.05, * * p<.01, * * * p<.001$ & & & &
\end{tabular}

Por último, respecto al consumo de combinados, presenta una única relación, levemente significativa, con la Actitud Evitativa ( $K=9.513, p=.049)$ (Tabla 5). Como ya se ha visto en los análisis descriptivos, la muestra de adolescentes tiene una Actitud Evitativa más alta que la población normal. También, y analizando los estadísticos del consumo de sustancias, eran los combinados la sustancia mayormente consumida por 
estos jóvenes. Parece, entonces, que el grado de evitación experiencial de los adolescentes puede tener algo que ver con la costumbre de beber copas los fines de semana como forma de emplear el tiempo de ocio.

Tabla 5. Diferencias en las creencias irracionales y aceptación en función del consumo de combinados

\begin{tabular}{llccccc}
\hline & Consumo & $N$ & $M$ & $D T$ & $K$ & Sig. \\
\hline \multirow{4}{*}{ AE } & Ninguno & 79 & 40.20 & 5.84 & & \\
& De 1 a 50 & 55 & 39.01 & 6.32 & & .049 \\
& De 51 a 100 & 38 & 38.05 & 5.93 & $9.513^{*}$ & \\
De 101 a 150 & 8 & 34.00 & 5.85 & & \\
Más de 150 & 20 & 39.90 & 8.34 & &
\end{tabular}

\section{DISCUSION}

Es interesante tomar en cuenta, como se verá más adelante, que ciertos fenómenos psicológicos van parejos a otros y puede que una modificación en una "parcela" mental contribuya a cambiar otra. Aplicado a las intervenciones, esto da mucha amplitud de técnicas y actividades para influir en la variable objetivo a modificar. Igualmente se rechazarán aquellos procedimientos que se sabe no serán eficaces por la inadecuada relación de contenidos.

El general, las puntuaciones del grupo de adolescentes de la muestra son similares al grupo de referencia poblacional en todas las variables. Las creencias irracionales muestran incluso una tendencia a la baja, sobre todo en Justificación de la Violencia. Sin embargo, hay un alto grado de acuerdo hacia ítems que hacen referencia al merecimiento de castigo. Las puntuaciones para el cuestionario de aceptación señalan un grado elevado de Evitación Experiencial, intermedio entre la evaluación normativa de los grupos clínico y no clínico. Respecto a la relación con el medio ambiente, en los jóvenes predomina un sentimiento claramente ecocéntrico y, por tanto, favorable a la protección y preocupación por medio ambiente.

En cuanto a las relaciones entre las creencias irracionales y la preocupación medioambiental, el antropocentrismo mantiene correlaciones positivas con la irracionalidad, en especial en aquellas dimensiones marcadas por una marcada preocupación por lo personal frente a los demás; mientras que en el ecocentrismo son negativas -excepto con la dimensión culpabilización; y es que las características del pensamiento ecocéntrico de armonía y compromiso con la Naturaleza, unido a la culpabilización de la sociedad por la situación de deterioro medioambiental, explican esa relación, en definitiva, marcada por una sensibilidad hacia la exigencia personal, más centrado en la motivación por la evitación del fracaso que por la consecución de éxitos. La sensibilidad hacia el bienestar y conservación de todos los seres vivos, que tan gran beneficio puede reportarles, se vuelve a veces en contra de la propia persona que se ve dominada por sentimientos que no puede controlar. Imagínese, como ejemplo no poco 
común, personas protectoras de los derechos de los animales a las que el sufrimiento de los mismos les lleva compromisos altruistas a la vez que a un sufrimiento empático propio que no pueden manejar.

En cuanto a la relación entre el antropocentrismo con la evitación de problemas y la necesidad de aceptación y éxito, no entra en contradicción con las características de la personalidad antropocéntrica. Se puede suponer el ejemplo de un sujeto que, guiado por sus necesidades de triunfo y éxito social, no repare en si los medios para alcanzar su estatus suponen o no un buen uso de los recursos medioambientales. Si sus aspiraciones entrasen en contradicción con un desarrollo sostenible y protección del ambiente, una opción sería el "mirar para otro lado" evitando el enfrentamiento con los problemas y, por tanto, adoptando una actitud bastante irracional. Parece que el antropocentrismo y las creencias irracionales pueden ir parejos en no pocas ocasiones.

Por otro lado, la relación que se produce entre las Creencias Irracionales y la Evitación Experiencial, en los que, aún perteneciendo a modelos terapéuticos distintos, comparten muchos rasgos en común. El punto de encuentro estaría ubicado más bien en aspectos emocionales. La dimensión evitativa Desbordamiento Emocional es la que más correlaciones significativas presenta con los grupos de creencias irracionales: Indefensión, Intolerancia a la Frustración y Necesidad de Aceptación y Éxito. En la misma línea, la correlación entre Desbordamiento emocional y Necesidad de Aceptación y Éxito es muy significativa, por lo que, de nuevo, se corrobora -con una terminología equivalente- el emparejamiento de la ansiedad con la necesidad de aceptación y éxito.

Por otra parte, dimensiones más cercanas al ámbito conductual, como la Respuesta ineficiente a los problemas vitales (del test de Aceptación) únicamente tiene una baja correlación significativa con la Culpabilización irracional. Las creencias de Justificación de la Violencia también se acercan más a la manifestación de un estilo conductual concreto, como es la puesta en marcha de acciones con marcado carácter agresivo. Tampoco en este caso se dan correlaciones con ningún grupo de respuestas de la prueba de Aceptación. Igual ocurre con la Evitación de Problemas y, en menor medida, con la Inadecuada Evaluación de los Problemas Vitales.

No existen relaciones significativas importantes entre la aceptación y la preocupación medioambiental. En cuanto a la relación de la Evitación experiencial con las Creencias Irracionales, se dan varias y significativas correlaciones entre sus subfactores, al contrario que la relación entre las creencias irracionales y la aceptación con el consumo de sustancias, en donde no hay un patrón claro de correlaciones -se dan índices significativos con algunas sustancias pero no con otras-.

Se obtienen diferencias estadísticamente significativas con el factor de creencias irracionales "indefensión". Una de las razones que pueden llevar a un adolescente a probar las drogas ilegales sería verse indefenso -con la sensación de no 
tener recursos para manejar un medio que le es "hostil"- frente a sus problemas cotidianos, la presión del grupo a consumir este tipo de drogas, o ambas cosas.

\section{REFERENCIAS}

Amérigo, M. y González, A. (2000). Los valores y las creencias medioambientales en relación con las decisiones sobre dilemas ecológicos. Estudios de Psicología, 22(1), 65-73.

Barraca, J. (2004). Spanish Adaptation of the Acceptance and Action Questionnaire (AAQ). International Journal of Psychology and Psychological Therapy, 4(3), 505-515.

Bruner, J.S. (1966). Toward a theory of instruction. Cambridge, Massachusetts: Harvard University Press. (Trad. Esp.: Uteha, 1972).

Camatta, C.D. y Nagoshi C.T. (1995). Stress, Depression, Irrational Beliefs, and Alcohol Use and Problems in a College Student Sample. Alcoholism: Clinical and Experimental Research, 19(1), 142-146.

Cardeñoso, O. y Calvete, E. (2004). Desarrollo de un "inventario de creencias irracionales" para adolescentes. Psicología Conductual, 12(2), 289-306.

Carrasco, I. (2005). Terapias racionales y de reestructuración cognitiva. En F.J. Labrador, J.A. Cruzado y M. Muñoz. Manual de técnicas de modificación y terapia de conducta (pp. 667-708) Madrid: Ediciones Pirámide.

Corral, A. y Pardo, P. (2001). Psicología evolutiva I. Volumen I. Introducción al desarrollo. Madrid: UNED.

Denoff, M.S. (2006). Drug abuse and alcoholism. Irrational beliefs as predictors of adolescent drug abuse and running away. Journal of Clinical Psychology, 43(3), 412-423.

Dunlap, R.E. y Van Liere, K.D. (1984). Commitment to the dominant social paradigm and concern for environmental quality. Social Science Quarterly, 65, 1013-1028.

Dunlap, R.E., Van Liere, K.D., Merting, A.G. y Jones, R.E. (2000). Measuring endorsement of the New Ecological Paradigm: A revised NEP escale. Journal of Social Issues, 56(3), 425-442.

Ellis, A. y Grieger, R. (1981). Manual de Terapia Racional-Emotiva. Bilbao: Desclée de Brouwer.

Erikson, E.H. (1980). Identidad, juventud y crisis. Madrid: Taurus. (Original de 1968).

González-Cabanach, R., Valle, A., Rodríguez, S., Piñeiro, I. y González, P. (2010). Las creencias motivacionales como factor protector del estrés en estudiantes universitarios. European Journal of Education and Psychology, 3(1), 75-87.

Hayes, S.C. (2004). Acceptance and commitment therapy, relational frame theory, and the third wave of behavioral and cognitive therapies. Behavior Therapy, 35, 639-665.

Hayes, S.C., Strosahl, K.D. y Wilson, K.G. (1999). Acceptance and commitment therapy. Nueva York: the Guilford Press.

Hayes, S.C., Wilson, K.G., Gifford, E.V., Follette, V.M. y Strosahl, K. (1996). Experiential avoidance and behavior disorder: A functional dimensional approach to diagnosis and treatment. Journal of Consulting and Clinical Psychology, 64, 1152-1168.

Hidalgo, M.I. y Redondo, A.M. (2005). Consumo de drogas en la adolescencia. Pediatría Integral IX (2), 137-155.

Holden, G.W., Moncher, M.S. y Schinke, S.P. (1990). Substance abuse. In A.S. Bellack, M. Hersen y A.E. Kazdin (Eds.) International handbook of behaviour modification and therapy, $2^{\text {nd }}$ ed. New York: Plenum.

Inhelder, B. y Piaget, J. (1955). De la lógica del niño a la lógica del adolescente. Buenos Aires. Paidós.

Kohlberg, L. (1984). Psicología del desarrollo moral. Bilbao: Desclée de Brouwer. 
Luciano, M.C. y Hayes, S.C. (2001). Trastorno de evitación experiencial. Revista Internacional de Psicología Clínica y de la Salud, 1, 109-157.

Luciano, M.C. y Valdivia, M.S. (2006). La Terapia de Aceptación y Compromiso (ACT). Fundamentos, características y evidencia. Papeles del Psicólogo, 27(2), 79-91.

Ministerio de Sanidad y Consumo (2006). Encuesta estatal sobre uso de drogas en enseñanzas secundarias (ESTUDES), 1994-2006. Madrid: Autor.

Pastor, Y., Balaguer, I. y García-Merita, M. (2006). Relaciones entre el autoconcepto y el estilo de vida saludable en la adolescencia media: un modelo exploratorio. Psicothema, 18(1), $18-24$.

Rosenberg, M. (1965). Society and the adolescent self-image. Princeton, NJ: Princeton University Press.

Vozmediano, L. y San Juan, C. (2005). Escala Nuevo Paradigma Ecológico: propiedades psicométricas con una muestra española obtenida a través de Internet. Medio Ambiente y Comportamiento Humano, 6(1), 37-49.

Recibido: 10 de febrero de 2010

Recepción Modificaciones: 19 de febrero de 2010

Aceptado: 22 de marzo de 2010 